\title{
Hole transport and photoluminescence in Mg-doped InN
}

\author{
N. Miller, ${ }^{1,2}$ J.W. Ager III, ${ }^{1, *}$ H.M. Smith III, ${ }^{1,2}$ M.A. Mayer, ${ }^{1,2}$ K.M. Yu, ${ }^{1}$ E.E. \\ Haller, ${ }^{1,2}$ W. Walukiewicz, ${ }^{1}$ W.J. Schaff, ${ }^{3}$ C. Gallinat, ${ }^{4}$ G. Koblmüller, ${ }^{4}$ and J.S. Speck ${ }^{4}$ \\ ${ }^{1}$ Materials Sciences Division, Lawrence Berkeley National Laboratory, Berkeley, CA, USA \\ ${ }^{2}$ Dept. of Materials Science and Engineering, \\ University of California - Berkeley, Berkeley, CA, USA \\ ${ }^{3}$ Dept. of Electrical and Computer Engineering, \\ Cornell University, Ithaca, NY, USA \\ ${ }^{4}$ Materials Department, University of California, Santa Barbara, CA, USA
}

(Dated: March 24, 2010)

\begin{abstract}
Hole conductivity and photoluminescence were studied in Mg-doped InN films grown by molecular beam epitaxy. Because surface electron accumulation interferes with carrier type determination by electrical measurements, the nature of the majority carriers in the bulk of the films was determined using thermopower measurements. Mg concentrations in a "window" from ca. $3 \times 10^{17}$ to $1 \times 10^{19} \mathrm{~cm}^{-3}$ produce hole-conducting, p-type films as evidenced by a positive Seebeck coefficient. This conclusion is supported by electrolyte-based capacitance voltage measurements and by changes in the overall mobility observed by Hall effect, both of which are consistent with a change from surface accumulation on an n-type film to surface inversion on a p-type film. The observed Seebeck coefficients are understood in terms of a parallel conduction model with contributions from surface and bulk regions. In partially compensated films with $\mathrm{Mg}$ concentrations below the window region, two peaks are observed in photoluminescence at $672 \mathrm{meV}$ and at $603 \mathrm{meV}$. They are attributed to band-to-band and band-to-acceptor transitions, respectively, and an acceptor binding energy of $\sim 70 \mathrm{meV}$ is deduced. In hole-conducting films with $\mathrm{Mg}$ concentrations in the window region, no photoluminescence is observed; this is attributed to electron trapping by deep states which are empty for Fermi levels close to the valence band edge.
\end{abstract}

\footnotetext{
${ }^{*}$ Corresponding author: JWAger@lbl.gov
} 


\section{INTRODUCTION}

Group III-nitride alloy band gaps are now known to span from the infrared ( $\mathrm{InN} \mathrm{E}_{g}=$ $0.675 \mathrm{eV}$ or more than $1800 \mathrm{~nm})$ to well into the ultraviolet $\left(\mathrm{GaN} \mathrm{E}_{g}=3.4 \mathrm{eV}\right.$ or $365 \mathrm{~nm}$, AlN $\mathrm{E}_{g}=6.1 \mathrm{eV}$ or $203 \mathrm{~nm}$ ), a range that includes not only the entire visible but virtually the entire solar spectrum $[1,2]$. Devices such as solar cells, laser diodes, and light emitting diodes made from group III-nitride alloys could therefore operate over this entire range of photon energies, making study of this alloy system of great importance.

InN has a very large electron affinity, and undoped InN films persistently exhibit n-type conductivity and a metallic surface accumulation layer due to pinning of the Fermi level above the conduction band edge [3-8]. Mg has been used as a p-type dopant, but the surface pinning in this case creates an n-type surface inversion layer which complicates Hall effect measurements and prevents direct electrical contact to any p-type material below. For this reason, simple single field Hall effect measurements on Mg-doped InN show all samples to be n-type. Electrolyte based capacitance-voltage (ECV) measurements have been used to verify the presence of ionizable acceptors in Mg-doped InN [9-13]. However, there are two limitations to this approach: 1) only the region within $\sim 10 \mathrm{~nm}$ of the surface is probed (due to high background concentration of donor defects), and 2) the measurement is sensitive to space charge and does not directly measure free carriers (holes) below the inversion layer.

Modeling of variable magnetic field Hall effect measurements using fields up to 12 Tesla is consistent with the presence of both electrons and holes in Mg-doped InN [14]. Noting that "hot probe" measurements can qualitatively distinguish between n-type and p-type In-rich InGaN samples [15], we suggest quantitative measurement of the Seebeck coefficient as a direct method to characterize hole transport and confirm p-type conductivity in Mg-doped InN. For the case of p-type InN, the primary advantage of a thermopower measurement over single field Hall effect measurement is that transport is induced through a temperature gradient, which pervades equally through the entire bulk of the sample while in a Hall effect measurement current flows due to an applied electric field (potential gradient), which drops predominantly within the metallic surface inversion layer of a p-InN sample. The Seebeck coefficient depends largely on the energy of carriers relative to the Fermi level as discussed further in section II. In general, the thermopower is larger for a sample with lower carrier concentration since the Fermi level is farther from the free carriers at the band 
edge. Similarly, thermopower increases with effective mass because increasing the mass increases the density of states, again increasing the Fermi-level/band-edge separation for a given carrier concentration. Both of these aspects favor detection of holes in the bulk rather than electrons at the surface of p-type InN since the electron concentration in the inversion layer is expected to be significantly larger than the bulk hole concentration and the hole effective mass in InN is predicted to be about 10 times larger than the electron effective mass $[16]$.

There are a few prior reports of positive Seebeck coefficients in Mg-doped InN $[13,17,18]$. Here, thermopower measurements are used to establish the range of $\mathrm{Mg}$ concentrations which lead to p-type conductivity. Contributions to the measured Seebeck coefficient from n-type regions (i.e., the film surface and interface with substrate/buffer layers below) are evaluated using a parallel conduction model. Photoluminescence (PL) in Mg-doped InN has been reported to disappear above a threshold $\mathrm{Mg}$ concentration $[9,14,19,20]$. Here, we show the threshold corresponds to the onset of hole conductivity, and the disappearance of PL is attributed to electron trapping by unoccupied defect states when the Fermi energy shifts towards the valence band edge.

\section{THEORY}

\section{A. Seebeck coefficient}

In the relaxation time approximation, the thermopower of electrons (an analogous expression holds for holes) can be written as follows

$$
S=\frac{-k_{\mathrm{B}}}{e}\left(\frac{\left\langle\mu_{k} \varepsilon / k_{\mathrm{B}} T\right\rangle}{\left\langle\mu_{k}\right\rangle}-\frac{\zeta}{k_{\mathrm{B}} T}\right)
$$

where $\mu_{k}$ is the $k$-dependent mobility defined below, $\varepsilon$ is the electron energy, and $\zeta$ is the Fermi energy defined to be zero at the conduction band edge [21-24]. Because of the nonparabolic band structure of InN, the mobility $\mu_{k}$ differs from the mobility of parabolic bands and is defined as $\mu_{k}=e \tau_{m} / m_{k}^{*}$ where $\tau_{m}$ is the momentum relaxation time and

$m_{k}$ is the $k$-dependent "momentum effective mass" $m^{*}(k)=\frac{\hbar^{2} k}{d E_{C}(k) / d k}$ where $E_{C}(k)$ is the conduction band dispersion relation. The thermal averages in the first term of Eq. (1) as a 
function of reduced energy $x=\varepsilon / k_{\mathrm{B}} T$ are defined as

$$
\langle\mu(x)\rangle=\int_{0}^{\infty}-\frac{\partial f_{o}(x, \eta)}{\partial x} \mu(x) k^{3}(x) d x
$$

where $f_{o}$ is the Fermi-Dirac distribution for the reduced Fermi energy $\eta=\zeta / k_{\mathrm{B}} T$ and $k(x)$ is the wavevector, which for electrons in $\mathrm{InN}$ follows a nonparabolic dispersion relation. Initial work with n-InN comparing electron concentrations obtained using Eq. (1) and $300 \mathrm{~K}$ measurements of $S$ with those from Hall effect have found good agreement [25]. Using Eq. (1), the thermopower of $\mathrm{n}$ - and p-type $\mathrm{InN}$ can be calculated as a function of carrier concentration as shown in Fig. 1. In this estimation we assume a nonparabolic conduction band with band edge electron effective mass of $0.065 \mathrm{mo}_{o}$, a parabolic valence band with hole effective mass of $0.64 m_{o}$, and only consider elastic scattering by assuming an energy power law dependence of the relaxation times of carriers $\left(\tau=A \varepsilon^{r}\right)$ where $r=1$ (as in reference [26]). More detailed calculations of $S$ for n-InN including both inelastic and elastic scattering will be reported separately [27].

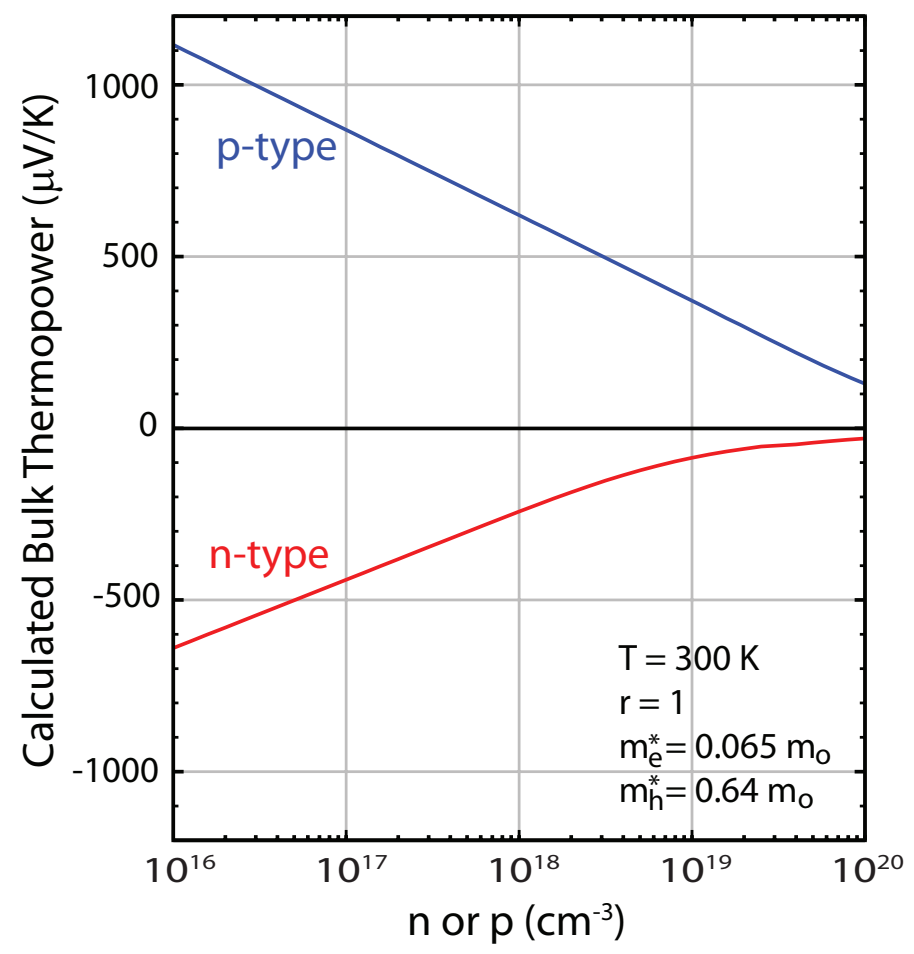

FIG. 1: Calculated carrier concentration dependence of the thermopower at $300 \mathrm{~K}$ of $\mathrm{n}$ - and p-type InN using Eq. (1).

Equation (1) can be used to calculate the temperature dependence of $S$ given that the carrier concentration and dominant scattering mechanisms are known. However, for strongly 
degenerate semiconductors, equation (1) reduces to the following relation [21],

$$
S_{\text {Degenerate }}=-\frac{k_{B}}{e}(r+3 / 2) \frac{\pi^{2}}{3} \frac{k_{B} T}{\zeta}
$$

where the terms have the same meaning as above. This form of the equation shows clearly that the Seebeck coefficient follows a simple linear temperature dependence for metallically doped semiconductors.

\section{B. Parallel conduction model}

The calculations of section II A are for homogeneous layers but, as discussed in section I, surface Fermi level pinning creates a metallic electron accumulation layer on n-type films and an n-type surface inversion layer on p-type films as shown schematically in Fig. 2. In addition, buffer or substrate layers under the InN films could also play a role.

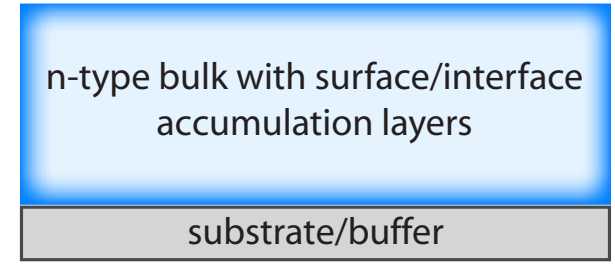

(a)

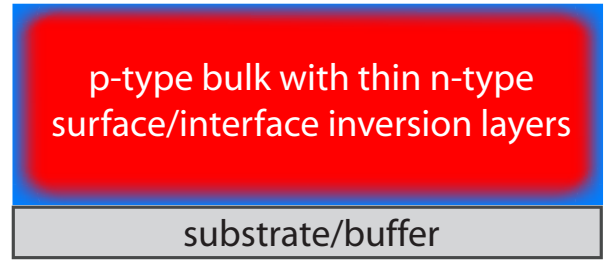

(b)

FIG. 2: (a) Illustration of n-type InN with surface and interface accumulation layers (blue), (b) illustration of p-type InN with p-type bulk (red) and surface/interface inversion layers (blue). The inversion and accumulation layer thicknesses are exaggerated here; in reality the surface layers are on the order of $10 \mathrm{~nm}$ thick while the films are on the order of 0.5 to $1 \mu \mathrm{m}$ thick.

The geometry of these experiments, in which thermopower is measured parallel to a shallow p-n junction, is uncommon, but has been considered before in the work of Baars et al. on $\mathrm{HgCdTe}(\mathrm{MCT})$ photodetectors and very recently in the work of Wagener et al. on p-type InAs [40-42]. The p-InAs study is especially relevant here given that InAs has similar defect properties to InN; the Fermi level is pinned above the conduction band edge at the surface, leading to surface inversion on p-type films and surface electron accumulation on n-type films [42-46]. In both the Baars and Wagener studies, a parallel conduction model is used to explain the observed thermopower of samples with buried p-n junctions parallel to the transport direction. 
Baars et al. use a parallel conduction model in which the observed Seebeck coefficient is given by a sum of the conductance-weighted-thermopower of each layer; for $j$ layers

$$
S_{\text {Observed }}=\sum_{j} S_{j} \frac{\sigma_{j} d_{j}}{\sigma_{T} d_{T}}
$$

where the total conductivity $\sigma_{T}=\sum_{j} \sigma_{j} \frac{d_{j}}{d_{T}}$ and the total thickness $d_{T}=\sum_{j} d_{j}$. In this approach all layers contribute to the observed thermopower as if connected in parallel. Since the thermopower of the thin surface inversion layer on p-InAs is very small in comparison to the bulk, Wagener et al. employ an approximation of this approach in which the observed Seebeck coefficient is simply given by the conductance-weighted-thermopower of the bulk: $S_{\text {Observed }}=S_{\text {Bulk }} \frac{\sigma_{B u l k} d_{\text {Bulk }}}{\sigma_{T} d_{T}}$.

In principle, potentials developed in the p-type bulk of the sample should be independent of those in the surface layer given that the two regions are separated by an insulating depletion layer. However, in the cases considered here, the junction isolation is not ideal. For example, MCT diodes can have a significant shunt current path provided by conducting dislocations $[47,48]$ and both InAs and InN thin films are typically grown on substrates for which there is a large lattice mismatch, leading to the formation of high dislocation densities. There is evidence that dislocations act as donors and may indeed act as junction shunts in these materials as well, providing a plausible explanation for why the depletion region fails to fully isolate the p-type bulk from the n-type surface layer [17, 27, 49-57].

It should be noted that any conducting layers will contribute to the thermopower of the sample including buffer/substrate layers. This is especially important when searching for p-type InN using thermopower measurements where the contribution of a conductive, ntype buffer layer could mask the positive thermopower of a p-type InN film. In this dataset, however, the contribution from substrate/buffer layers is assumed to be negligible because the films are grown on insulating GaN.

\section{EXPERIMENTAL}

InN thin film samples from Cornell University and the University of California - Santa Barbara (UCSB) were used for this work. The Cornell samples were grown by plasma assisted molecular beam epitaxy (PAMBE) on c-sapphire substrates using AlN nucleation and GaN buffer layers [28, 29]. The UCSB samples were grown by the same method on 
semi-insulating GaN commercial templates using 100 nm thick undoped GaN buffer layers [30, 31]. The composition and thickness of the films were determined by Rutherford Backscattering Spectrometry (RBS) measurements. RBS results showed that all of the films are stoichiometric (within an accuracy of $\sim 3 \%$ ). Channeling RBS (c-RBS) found minimum surface channeling yields of $<8 \%$, indicative of single crystal epitaxial films. The $\mathrm{Mg}$ content was measured by secondary ion mass spectrometry (SIMS) using Mg-implanted InN as a calibration standard.

For electrical and thermoelectric measurements, samples were cut into $5 \mathrm{~mm} \times 10 \mathrm{~mm}$ rectangles and In foil was pressed on to form Ohmic contacts. Thermopower (Seebeck coefficient) measurements were performed in the lateral gradient geometry by measuring the voltage that develops across a sample when a temperature gradient is applied. The Seebeck coefficient $S$ is given by the ratio $\Delta V / \Delta T$, where the deltas on voltage $V$ and temperature $T$ signify the difference between the values at the two ends of the sample. A more detailed description of the thermopower measurement system and procedure has been reported previously [13]. Hall effect measurements were performed with a 3000 Gauss magnet and In contacts in the van der Pauw configuration. Electrolyte-based capacitance voltage (ECV) measurements were performed with a Biorad ECV profiler using 1.0 M NaOH as the electrolyte. More details of our ECV procedures and analysis methods are reported elsewhere [9, 12, 13]. Photoluminescence measurements were performed using a SPEX 1680 $0.22 \mathrm{~m}$ double spectrometer and InSb photodiode detector cooled to $77 \mathrm{~K}$. PL spectra were corrected for instrument response using a calibrated lamp. The $515 \mathrm{~nm}$ line of an $\mathrm{Ar}$ ion laser was used as excitation source and PL was collected in the backscattering geometry. Samples were cooled to $\sim 25 \mathrm{~K}$ using a closed-cycle He cryostat.

\section{ELECTRICAL AND THERMOELECTRIC MEASUREMENTS}

\section{A. Results}

The measurement results for all the Mg-doped InN films are summarized in Table I, and the Seebeck coefficients measured from $\sim 150$ to $300 \mathrm{~K}$ are shown in Fig. 3a. Positive Seebeck coefficients are observed at room temperature for three of the samples (one sample has a positive $S$ at lower temperature). Because the Seebeck effect requires moving charge, this 
proves that there are positively charged carriers (holes) in the bulk of the films and that they are mobile, findings not easily obtained by other characterization techniques as discussed in section I. The range of room temperature Seebeck coefficients for all the samples extends from +868 to $-206 \mu \mathrm{V} / \mathrm{K}$; negative values indicate that electron transport is dominant.

TABLE I: Summary of electrical and thermoelectric data for Mg-doped InN at room temperature as measured by SIMS, Hall effect, and thermopower measurements. Sheet electron concentrations are reported since the presence of parallel conducting layers precludes a quantitative measurement of the bulk carrier concentration in some samples.

\begin{tabular}{lccccc}
\hline \hline Sample & Thickness $(\mathrm{nm})$ & $\mathrm{Mg}\left(\mathrm{cm}^{-3}\right)$ & $S(\mu \mathrm{V} / \mathrm{K})$ & {$[n]\left(\mathrm{cm}^{-2}\right)$} & $\mu\left(\mathrm{cm}^{2} / \mathrm{Vs}\right)$ \\
\hline GS1548 & 445 & $4 \times 10^{19}$ & -54 & $9.8 \times 10^{13}$ & 22 \\
GS1547 & 480 & $2 \times 10^{19}$ & -27 & $9.7 \times 10^{13}$ & 42 \\
GS1810 & 450 & $6 \times 10^{18}$ & 74 & $1.2 \times 10^{14}$ & 25 \\
GS1650 & 1290 & $8 \times 10^{18}$ & 386 & $2.0 \times 10^{14}$ & 11 \\
$101107 \mathrm{~A}$ & 820 & $2 \times 10^{18}$ & 868 & $2.9 \times 10^{14}$ & 18 \\
$101107 \mathrm{~B}$ & 925 & $3 \times 10^{17}$ & -2 & $1.2 \times 10^{14}$ & 264 \\
$101107 \mathrm{C}$ & 950 & $3 \times 10^{16}$ & -206 & $4.0 \times 10^{13}$ & 1554 \\
\hline \hline
\end{tabular}

The room temperature Seebeck coefficient as a function of Mg content measured by SIMS is shown in Fig. 3b. The Seebeck coefficient depends non-monotonically on the $\mathrm{Mg}$ content of the film. Over the range of $\mathrm{Mg}$ concentrations in this study, $S$ is first negative, then increases with increasing $\mathrm{Mg}$ content to a large positive value, then eventually decreases to negative values again, implying that there is a "window" of $\mathrm{Mg}$ content that leads to p-type conductivity.

ECV data for the Mg-doped InN samples studied here are shown in Fig. 4. The dashed line is a guide for the eye showing that the peak shifts to lower applied bias for samples with Mg concentration above some threshold, which corresponds well with the transition from negative to positive thermopower. 


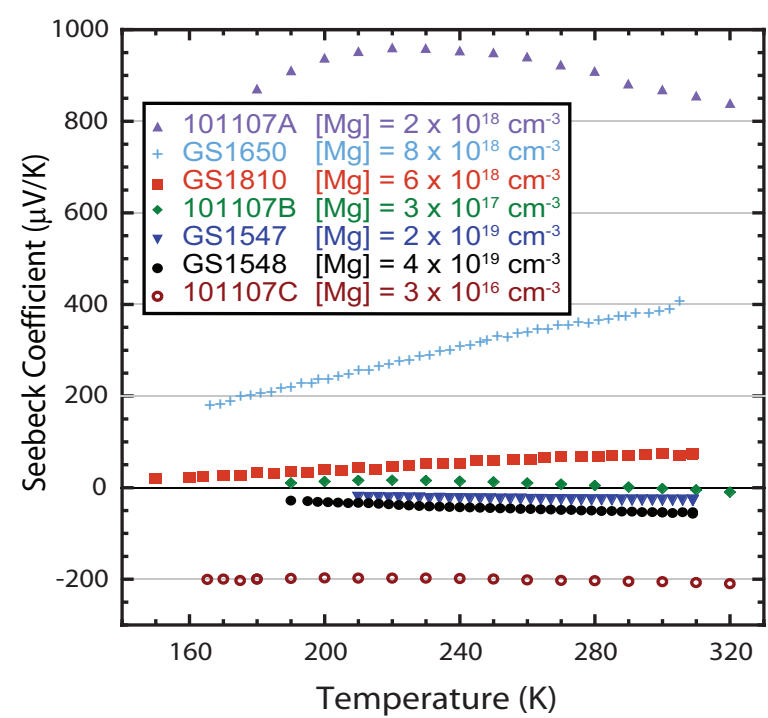

(a)

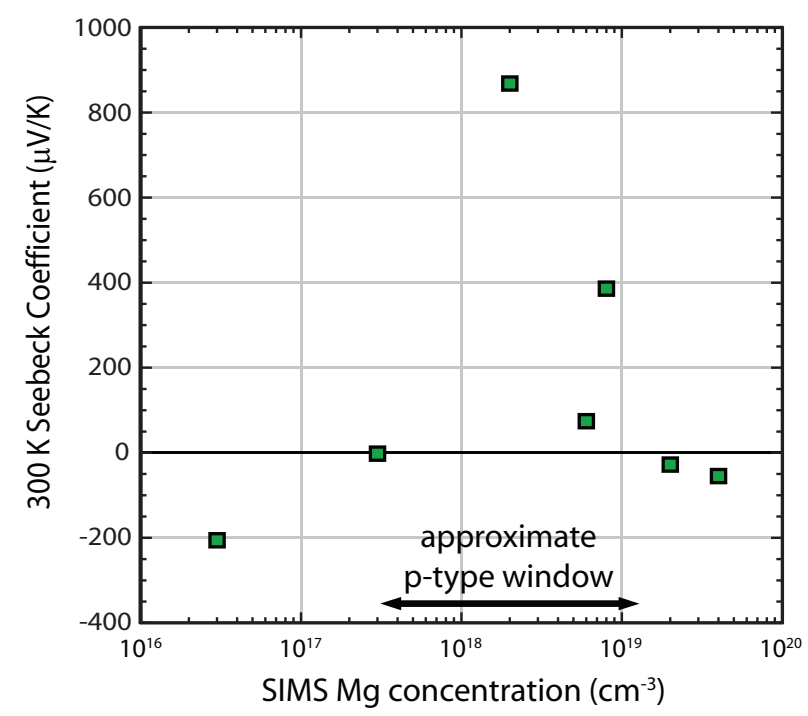

(b)

FIG. 3: a) Measured Seebeck coefficient of Mg-doped InN samples as a function of temperature. SIMS Mg content shown in legend. b) Measured Seebeck coefficient of Mg-doped InN samples at room temperature as a function of the Mg concentration measured by SIMS.

\section{B. Discussion}

Too little $\mathrm{Mg}$ or too much can lead to n-type conductivity, consistent with reports based on ECV measurements that a Mg concentration in the range $\sim 10^{18}$ to $\sim 10^{20} \mathrm{~cm}^{-3}$ results in a net-concentration of acceptors $\left(N_{A}-N_{D}>0\right)$ but a $\mathrm{Mg}$ concentration outside this range results in a net-concentration of donors in $\mathrm{InN}$ [11]. These stages are illustrated in Fig. 2. Undoped films, or those with $\mathrm{Mg}$ concentration less than $\sim 3 \times 10^{17} \mathrm{~cm}^{-3}$, are n-type throughout with a surface accumulation layer as shown in Fig. 2a. With sufficient Mg doping, $\gtrsim 3 \times 10^{17} \mathrm{~cm}^{-3}$, the films become p-type in the bulk but retain the surface and interface inversion layers as shown in Fig. 2b. With too much $\mathrm{Mg}$ doping (high $10^{18} \mathrm{~cm}^{-3}$ range) compensating donors begin to form until eventually at $\mathrm{Mg}$ concentrations $\gtrsim 10^{20} \mathrm{~cm}^{-3}$ fully n-type films result again as in Fig. 2a, but with higher bulk electron concentrations.

The Mg-doping trend shown in Fig. 3b is consistent with the ECV and Hall data. Recent experimental and theoretical reports on ECV measurements of Mg-doped InN have shown that the voltage at which the minimum capacitance occurs (maximum in $C^{-2}$ vs. $V$ "Mott-Schottky" plots) is indicative of the net charge type (donor or acceptor) beneath the surface inversion/accumulation layer [10, 11]. This "turnover" occurs at lower applied bias 


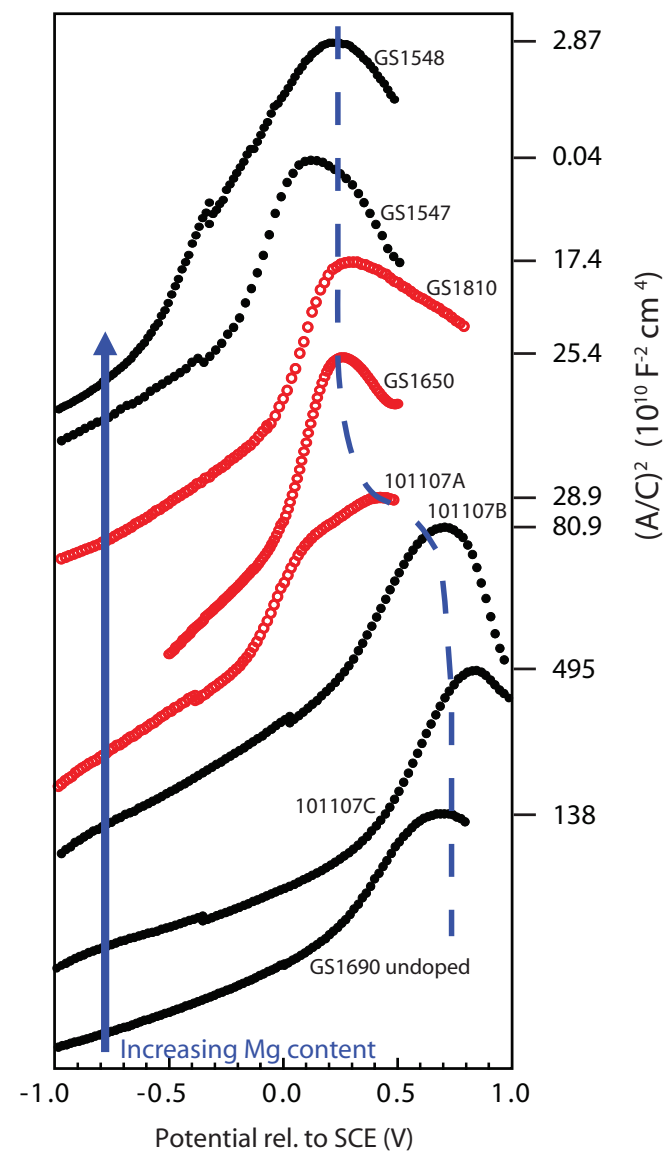

FIG. 4: Mott-Schottky plot (area divided by capacitance, the quantity squared vs. voltage, plotted here as potential relative to a standard calomel electrode (SCE)) showing all of the Mg-doped samples and one undoped sample (GS1690, $n=1.2 \times 10^{18} \mathrm{~cm}^{-3}$ ) for reference. The curves are each plotted on their own log scale and vertically offset for clarity; the hashes on the right hand side mark the maximum for each curve. Samples with positive (negative) Seebeck coefficients at room temperature are shown in red (black) with open (closed) symbols. The blue dashed line is a guide for the eye.

in samples with a net-concentration of acceptors below the surface, and at higher applied bias in samples with a net-concentration of donors below the surface. Examining Fig. 4, one sees that the films with $\mathrm{Mg}$ concentration $\geq 2 \times 10^{18} \mathrm{~cm}^{-3}$ all show slope inversion at lower bias voltages, consistent with a net-concentration of acceptors below the surface. The samples with $\mathrm{Mg}$ concentration $\leq 3 \times 10^{17} \mathrm{~cm}^{-3}$ exhibit curves that turn over at relatively higher bias, similar to the n-type, undoped sample also shown for reference in Fig. 4.

In the work of Wang et al., the turnover voltage returns to higher voltages for the heaviest 
Mg-doped films indicating a return to fully n-type material, which is not observed in this study, presumably because the highest $\mathrm{Mg}$ content film in this study $\left([M g]=4 \times 10^{19}\right.$ $\mathrm{cm}^{-3}$ ) is below the threshold for complete donor compensation reported to be $\sim 10^{20} \mathrm{~cm}^{-3}$ [11]. The ECV results of samples studied here show that there is still a net-concentration of acceptors in the near surface region for the samples with highest $\mathrm{Mg}$ content, but do not rule out the possibility of a net-concentration of donors, and therefore regions of n-type conductivity, deeper within the films.

Considering the experimental data as a whole, we can make conclusions about the $\mathrm{Mg}$ doping dependence. Incorporation of too little $\mathrm{Mg}$ fails to overcome the background electron concentration $n_{\text {min }}$, which is typically $\sim 3 \times 10^{17} \mathrm{~cm}^{-3}$ in InN, resulting in n-type conductivity with increased compensation. This assertion is corroborated by the electrical and thermoelectric data shown in Table I; sample $101107 \mathrm{C}$ with Mg concentration below $n_{\min }$ is a partially compensated film with low (for InN) carrier concentration, high mobility, and large negative Seebeck coefficient, but sample 101107B with Mg concentration approaching $n_{\min }$ is closely compensated with greater carrier concentration, much lower mobility, and near-zero room temperature Seebeck coefficient. For samples with more Mg, mobility is even lower, consistent with the interpretation that only surface electron transport is contributing to the Hall effect results as discussed previously in section I [9].

The incorporation of $\mathrm{Mg}$ at $\gtrsim 10^{19} \mathrm{~cm}^{-3}$ results again in InN films with negative $S$, indicating dominant electron transport. There are several possible sources of compensating donors in InN films with high $\mathrm{Mg}$ concentrations. In GaN, overdoping with $\mathrm{Mg}$ has been shown to produce donors and reduce the free hole concentration; this result has been attributed to the formation of compensating defect complexes ( $\operatorname{such}$ as $\mathrm{Mg}_{\mathrm{N}} \mathrm{V}_{N}$ ) $[32,33]$ and pyramidal inversion domains [34, 35]. TEM studies of Mg-doped InN, InGaN, and GaN have shown that high levels of Mg result in large densities of planar extended defects, which could also be contributing to the n-type conductivity of overdoped films [36-39].

With two notable exceptions, the temperature dependence of $|S|$ plotted in Fig. 3a is generally linear and decreasing with decreasing temperature, consistent with metallically doped semiconductor behavior as discussed in section II A. However, the two samples with Mg content at or slightly above $n_{\text {min }}$, samples 101107A and 101107B, do have nonlinear $S$ vs. $T$ curves. The Seebeck coefficient of sample $101107 \mathrm{~B}$ is slightly negative at room temperature but crosses through zero and becomes positive below $\sim 280 \mathrm{~K}$, only to reach a 
maximum at $\sim 220 \mathrm{~K}$ before decreasing again. Similarly, the Seebeck coefficient of sample 101107A increases upon cooling, reaches a maximum near $220 \mathrm{~K}$, then decreases upon further cooling. With contributions to the observed Seebeck coefficient coming from both n-type and p-type conducting regions, this temperature dependence may be analyzed in terms of the parallel conduction model of section II B. In n-type samples, variable temperature Hall effect measurements have shown that electron concentration is nearly temperature invariant; mobility also often depends weakly on temperature except in samples with very low electron concentrations, which exhibit the typical inverted U-shape due to phonon scattering and ionized impurity scattering at high and low temperatures, respectively $[17,27,54,58]$. If the electron mobility in n-type regions were increasing over this temperature range it would be expected to decrease the observed Seebeck coefficient rather than increase it. Thus, the temperature dependence of the Seebeck coefficient observed in these two samples likely reflects changes in hole conductivity rather than electron conductivity.

Using Eq. (4) and the Seebeck coefficients estimated in section II, the competing contributions of electron and hole conducting layers can be evaluated. In an otherwise bulk p-type InN sample, as in the case shown in Fig. 2b, similar, highly degenerate n-type inversion layers are expected on the surface as well as at the InN/substrate or InN/buffer-layer interface [3-8]. Because these layers are degenerate $\left(n \sim 10^{20} \mathrm{~cm}^{-3}\right)[4,6]$, their Seebeck coefficients are on the order of tens of $\mu \mathrm{V} / \mathrm{K}$ as shown in Fig. 1. And due to their close proximity to the surface, mobility of inversion layer carriers is expected to be low; if single-field Hall measurements of p-type samples are indeed dominated by inversion/interface electron transport, then conductivity is on the order of only 1-10 $(\Omega-\mathrm{cm})^{-1}$. For comparison, the bulk p-type free hole concentration is likely lower $\left(p \sim 10^{17}-10^{19} \mathrm{~cm}^{-3}\right)$, yielding larger Seebeck coefficients $(\sim 400-800 \mu \mathrm{V} / \mathrm{K})$ as shown in Fig. 1, and hole mobility has been estimated in the range of 17 to $36 \mathrm{~cm}^{2} / \mathrm{Vs}$ [59]. Now examining Eq. (4) and considering further the factor of $\sim 100$ difference in thickness between the inversion layers and the bulk p-type material, it is clear that very bulk-like positive Seebeck coefficients are expected when the only electron transport contribution comes from inversion layers. This effect is easily illustrated with an example: a $500 \mathrm{~nm}$ thick p-type film with free hole concentration $p=10^{18} \mathrm{~cm}^{-3}$ and hole mobility $\mu_{h}=30 \mathrm{~cm}^{2} / \mathrm{Vs}$ would have a Seebeck coefficient of approximately $+600 \mu \mathrm{V} / \mathrm{K}$, but adding the contribution of converting $10 \mathrm{~nm}$ of this film to n-type material with conductivity $10(\Omega-\mathrm{cm})^{-1}$ and $S=-40 \mu \mathrm{V} / \mathrm{K}$ only reduces the observed Seebeck coefficient to 
$S_{\text {Observed }} \simeq 574 \mu \mathrm{V} / \mathrm{K}$ or within $96 \%$ of the bulk value. And indeed, large, positive Seebeck coefficients, similar to those predicted by theory, are measured for samples 101107A and GS1650 which have Mg doping in the "window" range.

In contrast, the samples with more Mg (e.g. GS1547 and GS1548) have small, negative Seebeck coefficients, suggesting an electron contribution in addition to that of the inversion layers to explain the apparent dominance of n-type conductivity in the thermopower measurements. In fact it has been shown for sample GS1548 (the Mg-doped sample of reference [60]) that even when the surface inversion layer is depleted with an electrolyte, an n-type Hall effect is still measured with a sheet concentration of $\sim 10^{14} \mathrm{~cm}^{-2}$. This indicates the presence of a thicker layer of n-type conducting material, likely due to the combined effects of self-compensation and increased extended defect density with large Mg concentrations as well as interface-related charge $[6,61]$. Using Eq. (4), we note that the contribution of a thicker, more conductive bulk-like n-type layer, in addition to the inversion layers and the bulk p-type layer, can drastically reduce the observed Seebeck coefficient. This effect is easily illustrated with an example: a $500 \mathrm{~nm}$ thick p-type film with free hole concentration $p=10^{18} \mathrm{~cm}^{-3}$ and hole mobility $\mu_{h}=30 \mathrm{~cm}^{2} /$ Vs would have a Seebeck coefficient of approximately $+600 \mu \mathrm{V} / \mathrm{K}$, but adding the contribution of just $30 \mathrm{~nm}$ of n-type material with free electron concentration $n=10^{19} \mathrm{~cm}^{-3}$, electron mobility $\mu_{e}=300 \mathrm{~cm}^{2} / \mathrm{Vs}$, and $S=-100 \mu \mathrm{V} / \mathrm{K}$ is enough to reduce the observed Seebeck coefficient to negative values ( $S_{\text {Observed }} \simeq-5 \mu \mathrm{V} / \mathrm{K}$ in this case).

Thus, parallel conduction modeling of Mg-doped InN samples is consistent with thermopower, Hall effect, and capacitance-voltage measurements and electrolyte-gated Hall results reported in reference [60]. This modeling shows that the contribution of inversion layers alone to the observed Seebeck coefficient can be minor, but the presence of high conductivity n-type material in excess of the normal inversion layers can substantially reduce the observed Seebeck coefficient, even to negative values, with only a fraction of the total film thickness represented as n-type material.

\section{PHOTOLUMINESCENCE}

Photoluminescence is observed only from the two samples with the lowest Mg concentration, consistent with previous reports of PL being quenched by Mg doping [14, 19, 20]. A 
plausible interpretation of this behavior is that trap states in the bulk of the film are emptied as the Fermi level drops, opening up nonradiative recombination paths for the photoexcited carriers. This view is supported indirectly by the observation that $\mathrm{PL}$ is quenched for $\mathrm{Mg}$ concentrations roughly greater than or equal to the residual donor concentration $[14,19]$. This view is also supported by the recovery of PL in InN:Mg by irradiation sufficient to compensate the free hole concentration [9]. Fig. 5a shows PL spectra of the sample with the lowest Mg content (101107C) for three different levels of laser excitation intensity. Two peaks are clearly resolved, one near the InN bandgap energy at $672 \mathrm{meV}$, which is attributed to band-to-band transitions (labeled $b b$ ), and one $\sim 70 \mathrm{meV}$ lower at $603 \mathrm{meV}$ attributed to band-to-acceptor transitions (labeled $b a$ ) as discussed below.

In addition to band-to-band emission, a Mg-related peak has been observed in PL spectra of InN films grown by both MBE and metal organic chemical vapor deposition; in both cases the Mg-related peak appeared at an energy $\sim 60 \mathrm{meV}$ below the band-to-band emission and was interpreted as emission from band-edge electrons recombining with holes at the $\mathrm{Mg}$ acceptor state $\sim 60 \mathrm{meV}$ above the valence band edge $[19,20]$. In another report, a single PL peak was observed in InN:Mg 110 meV lower than in other samples with less $\mathrm{Mg}$, leading to a larger estimate of $\sim 110 \mathrm{meV}$ for the $\mathrm{Mg}$ activation energy [14]. Transitions to acceptor levels have also been reported in detailed studies of PL in undoped InN [62, 63]. In the study by Arnaudov et al., two PL peaks are observed, which are attributed to transitions of band-edge electrons to two different acceptor states with ionization energies of $18 \mathrm{meV}$ and $85 \mathrm{meV}$, respectively. In the study by Klochikhin et al., again two PL peaks are observed; the higher energy peak is attributed to a combination of band-to-band transitions and transitions of band-edge electrons to Urbach tails in the valence band and/or to a shallow acceptor with ionization energy 5-10 meV, while the lower energy peak is attributed to transitions of band-edge electrons to a deeper acceptor with ionization energy of 50-55 $\mathrm{meV}$. Nearly all of these reports are consistent with a hydrogenic acceptor in InN since the hole effective mass is not well known; assuming it falls in the range $m_{h}^{*}=0.42-0.7 m_{\text {。 }}$ and using $\varepsilon_{\circ}=10.3$ as the static dielectric constant [64], the acceptor ionization energy is estimated to be in the range of $54-90 \mathrm{meV}$.

In the context of these previous reports, the $b b$ peak in Fig. 5a is attributed to bandto-band emission, while the $b a$ peak is attributed to transitions of free electrons near the conduction band edge to the $\mathrm{Mg}$ acceptor state located $\sim 70 \mathrm{meV}$ above the valence band 


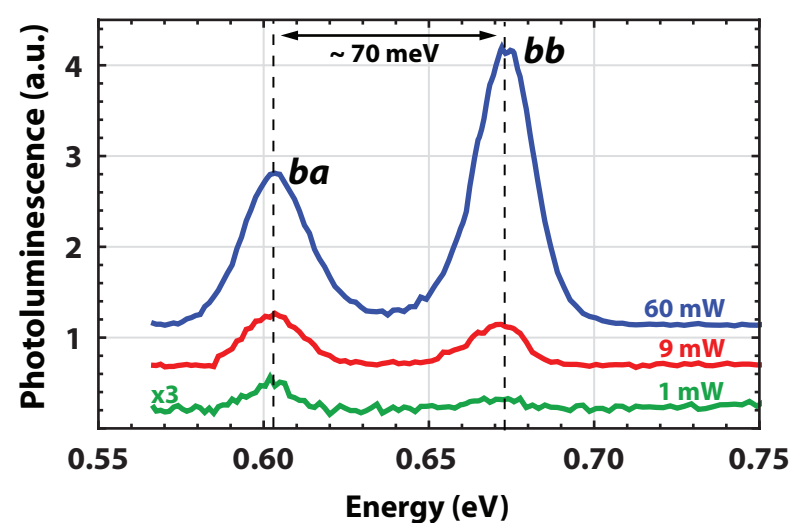

(a)

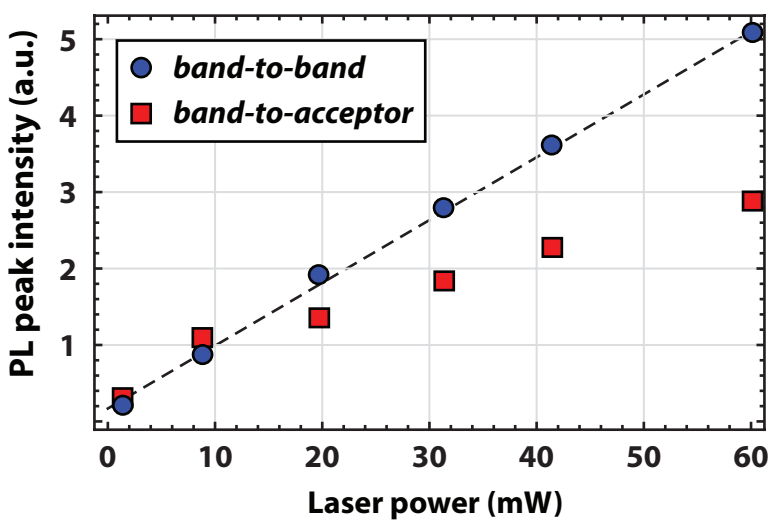

(b)

FIG. 5: (a) Photoluminescence spectra at $~ 25 \mathrm{~K}$ of the sample with the lowest Mg content, 101107C $\left(S_{300 \mathrm{~K}}=-206 \mu \mathrm{V} / \mathrm{K}\right)$, for several different laser excitation intensities, vertically offset for clarity. Two peaks are clearly resolved: one associated with band-to-band emission $(b b)$, the other with band-to-acceptor transitions $(b a)$ as explained in the text. (b) Peak PL intensity is plotted as a function of pumping laser power. The dashed line in (b) is a linear fit to the $b b$ data.

edge. Further evidence for this interpretation comes from the dependence of the relative intensities of these two peaks on pumping intensity. As shown in Fig. 5b, the dominant emission line at low excitation intensities is the $b a$ peak; with increasing laser intensity the $b b$ peak increases linearly and becomes the dominant emission line while the $b a$ peak increases more slowly, in excellent agreement with the findings reported by Wang et al. [19]. One interpretation of this behavior, which has been observed before in both Mg-doped and undoped InN, is that the majority of photoholes are localized on acceptor sites for low laser intensity but with increasing laser intensity the photohole population increases, the quasi Fermi level of holes moves towards the valence band, and more photoholes begin to occupy delocalized valence band states, leading to increasing band-to-band emission and decreasing band-to-acceptor emission [19, 63].

\section{CONCLUSIONS}

$\mathrm{Mg}$ concentrations in a "window" from $\sim 3 \times 10^{17}$ to $\sim 1 \times 10^{19} \mathrm{~cm}^{-3}$ produce p-type InN films as evidenced by a positive Seebeck coefficient. This conclusion is supported by changes in mobility observed by Hall effect and by capacitance voltage measurements, both 
of which are consistent with a change from surface accumulation on an n-type film to surface inversion on a p-type film. A parallel conduction path model is used to model the dependence of the observed thermopower on the properties of the films. At Mg concentrations below the window region, two peaks are observed in photoluminescence at $672 \mathrm{meV}$ and $603 \mathrm{meV}$, which are attributed to band-to-band and band-to-acceptor transitions, respectively, and an acceptor binding energy of $\sim 70 \mathrm{meV}$ is deduced. At $\mathrm{Mg}$ concentrations in the window region, no PL is observed; this is attributed to electron trapping in deep states which become empty as the Fermi level drops below mid-gap.

\section{Acknowledgments}

The authors acknowledge D. Lefforge of Evans Analytical Group for SIMS measurements, R. Lieten for help with x-ray diffraction measurements, L. Reichertz and J. Beeman for help with instrumentation and sample processing, R. Broesler for discussions of PL, Z. LilientalWeber for discussions of Mg-related defects in nitrides, and T. D. Veal and P. D. C. King for useful discussions of surface states, Mg doping, and SIMS. This work was supported by the Director, Office of Science, Office of Basic Energy Sciences, Division of Materials Sciences and Engineering, of the U.S. Department of Energy under Contract No. DE-AC0205CH11231. N. M. and M. M. also thank the U.S. Department of Defense for fellowship support under the NDSEG program.

[1] Schley, P., Goldhahn, R., Gobsch, G., Feneberg, M., Thonke, K., Wang, X., and Yoshikawa, A. Phys. Status Solidi B 246, 1177 (2009).

[2] Wu, J., Walukiewicz, W., Yu, K. M., Shan, W., Ager III, J. W., Haller, E. E., Lu, H., Schaff, W. J., Metzger, W. K., and Kurtz, S. J. Appl. Phys. 94, 6477 (2003).

[3] Lu, H., Schaff, W. J., Eastman, L. F., and Stutz, C. E. Appl. Phys. Lett. 82, 1736 (2003).

[4] Mahboob, I., Veal, T. D., McConville, C. F., Lu, H., and Schaff, W. J. Phys. Rev. Lett. 92, 036804 (2004).

[5] Li, S. X., Yu, K. M., Wu, J., Jones, R. E., Walukiewicz, W., Ager III, J. W., Shan, W., Haller, E. E., Lu, H., and Schaff, W. J. Phys. Rev. B 71(16), 161201 (2005). 
[6] Veal, T. D., Piper, L. F. J., Mahboob, I., Lu, H., Schaff, W. J., and McConville, C. F. Physica Status Solidi C 2, 2246-2249 (2005).

[7] Cimalla, V., Niebelschütz, M., Ecke, G., Lebedev, V., Ambacher, O., Himmerlich, M., Krischok, S., Schaefer, J. A., Lu, H., and Schaff, W. J. Physica Status Solidi A 203(1), 59-65 (2006).

[8] Klochikhin, A. A., Davydov, V. Y., Strashkova, I. Y., and Gwo, S. Physical Review B 76(23), 235325 (2007).

[9] Jones, R. E., Yu, K. M., Li, S. X., Walukiewicz, W., Ager III, J. W., Haller, E. E., Lu, H., and Schaff, W. J. Phys. Rev. Lett. 96(12), 125505 (2006).

[10] Yim, J. W. L., Jones, R. E., Yu, K. M., Ager III, J. W., Walukiewicz, W., Schaff, W. J., and Wu, J. Phys. Rev. B 76(4), 41303 (2007).

[11] Wang, X., Che, S. B., Ishitani, Y., and Yoshikawa, A. Appl. Phys. Lett. 91, 242111 (2007).

[12] Ager III, J. W., Jones, R. E., Yamaguchi, D. M., Yu, K. M., Walukiewicz, W., Li, S. X., Haller, E. E., Lu, H., and Schaff, W. J. Phys. Status Solidi B 244(6), 1820 (2007).

[13] Ager III, J. W., Miller, N., Jones, R. E., Yu, K. M., Wu, J., Schaff, W. J., and Walukiewicz, W. Phys. Status Solidi B $\mathbf{2 4 5 ( 5 ) , ~} 873$ (2008).

[14] Anderson, P. A., Swartz, C. H., Carder, D., Reeves, R. J., Durbin, S. M., Chandril, S., and Myers, T. H. Appl. Phys. Lett. 89, 184104 (2006).

[15] Matthews, K. D., Chen, X., Hao, D., Schaff, W. J., and Eastman, L. F. Phys. Status Solidi C 5, 1863-1865 (2008).

[16] Veal, T. D., Piper, L. F. J., Schaff, W. J., and McConville, C. F. J. Cryst. Growth 288(2), 268-272 (2006).

[17] Miller, N., Ager III, J. W., Jones, R. E., Smith III, H. M., Mayer, M. A., Yu, K. M., Hawkridge, M. E., Liliental-Weber, Z., Haller, E. E., Walukiewicz, W., Schaff, W. J., Gallinat, C., Koblmüller, G., and Speck, J. S. Physica B 404, 4862-4865 (2009).

[18] Dmowski, L. H., Baj, M., Suski, T., Przybytek, J., Czernecki, R., Wang, X., Yoshikawa, A., Lu, H., Schaff, W. J., Muto, D., and Nanishi, Y. Journal of Applied Physics 105(12), 123713 (2009).

[19] Wang, X., Che, S. B., Ishitani, Y., and Yoshikawa, A. Appl. Phys. Lett. 90, 201913 (2007).

[20] Khan, N., Nepal, N., Sedhain, A., Lin, J. Y., and Jiang, H. X. Appl. Phys. Lett. 91, 012101 (2007). 
[21] Seeger, K. Semiconductor physics. Springer New York, (2002).

[22] Nolas, G. S., Sharp, J., and Goldsmid, H. J. Thermoelectrics: Basic Principles and New Materials Developments. Springer, (2001).

[23] Kolodziejczak, J. and Zukotynski, S. Phys. Status Solidi B 5(1) (1964).

[24] MacDonald, D. K. C. Thermoelectricity: an introduction to the principles. John Wiley and Sons, Inc., New York, (1962).

[25] Miller, N. Master's thesis, University of California - Berkeley, (2009).

[26] Jones, R. E., Li, S. X., Hsu, L., Yu, K. M., Walukiewicz, W., Liliental-Weber, Z., Ager III, J. W., Haller, E. E., Lu, H., and Schaff, W. J. Physica B: Physics of Condensed Matter 376, $436(2006)$.

[27] Miller, N., Ager III, J. W., Jones, R. E., Smith III, H. M., Yu, K. M., Hawkridge, M. E., Haller, E. E., Walukiewicz, W., Schaff, W. J., Gallinat, C., Koblmüller, G., and Speck, J. S. unpublished .

[28] Lu, H., Schaff, W. J., Hwang, J., Wu, H., Yeo, W., Pharkya, A., and Eastman, L. F. Appl. Phys. Lett. 77(16), 2548 (2000).

[29] Lu, H., Schaff, W. J., Hwang, J., Wu, H., Koley, G., and Eastman, L. F. Appl. Phys. Lett. 79, 1489 (2001).

[30] Gallinat, C. S., Koblmüller, G., Brown, J. S., and Speck, J. S. J. Appl. Phys. 102(6), 064907 (2007).

[31] Koblmüller, G., Gallinat, C. S., and Speck, J. S. J. Appl. Phys. 101, 083516 (2007).

[32] Kaufmann, U., Schlotter, P., Obloh, H., Köhler, K., and Maier, M. Phys. Rev. B 62(16), 10867 (2000).

[33] Romano, L. T., Kneissl, M., Northrup, J. E., Van de Walle, C. G., and Treat, D. W. Applied Physics Letters 79(17), 2734-2736 (2001).

[34] Vennéguès, P., Leroux, M., Dalmasso, S., Benaissa, M., De Mierry, P., Lorenzini, P., Damilano, B., Beaumont, B., Massies, J., and Gibart, P. Phys. Rev. B 68(23), 235214 Dec (2003).

[35] Liliental-Weber, Z., Tomaszewicz, T., Zakharov, D., Jasinski, J., and O'Keefe, M. A. Phys. Rev. Lett. 93, 206102 (2004).

[36] Liliental-Weber, Z. Indium Nitride and Related Alloys, chapter 14: Structure of InN and their alloys with Ga; Transmission Electron Microscopy Studies. CRC Press, Taylor and Francis Group, LLC (2009). 
[37] Liliental-Weber, Z., Benamara, M., Swider, W., Washburn, J., Grzegory, I., Porowski, S., Lambert, D. J. H., Eiting, C. J., and Dupuis, R. D. Appl. Phys. Lett. 75(26), 4159-4161 (1999).

[38] Wang, X., Che, S.-B., Ishitani, Y., Yoshikawa, A., Sasaki, H., Shinagawa, T., and Yoshida, S. Appl. Phys. Lett. 91(8), 081912 (2007).

[39] Pezzagna, S., Venngus, P., Grandjean, N., and Massies, J. J. Cryst. Growth 269(2-4), 249 $256(2004)$.

[40] Baars, J., Brink, D., Edwall, D. D., and Bubulac, L. O. J. Electron. Mater. 22(8), 923-929 (1993).

[41] Baars, J., Brink, D., and Ziegler, J. Journal of Vacuum Science \& Technology B: Microelectronics and Nanometer Structures 9, 1709 (1991).

[42] Wagener, M. C., Wagener, V., and Botha, J. R. Applied Physics Letters 94, 262106 (2009).

[43] Gopal, V., Chen, E. H., Kvam, E. P., and Woodall, J. M. Journal of Electronic Materials 29(11), 1333-1339 (2000).

[44] Piper, L. F. J., Veal, T. D., Lowe, M. J., and McConville, C. F. Physical Review B 73(19), $195321(2006)$.

[45] Olsson, L., Andersson, C. B. M., Håkansson, M. C., Kanski, J., Ilver, L., and Karlsson, U. O. Physical review letters 76(19), 3626-3629 (1996).

[46] Smit, K., Koenders, L., and Mönch, W. Journal of Vacuum Science E Technology B: Microelectronics and Nanometer Structures 7, 888 (1989).

[47] Johnson, S. M., Rhiger, D. R., Rosbeck, J. P., Peterson, J. M., Taylor, S. M., and Boyd, M. E. Journal of Vacuum Science $\&$ Technology B: Microelectronics and Nanometer Structures 10, 1499 (1992).

[48] Baker, I. M. and Maxey, C. D. J. Electron. Mater. 30(6), 682-689 (2001).

[49] Tsukamoto, H., Chen, E.-H., Woodall, J. M., and Gopal, V. Applied Physics Letters 78(7), 952-954 (2001).

[50] Piper, L. F. J., Veal, T. D., McConville, C. F., Lu, H., and Schaff, W. J. Appl. Phys. Lett. 88, 252109 (2006).

[51] Takei, Y. and Nakayama, T. J. Cryst. Growth 311, 2767 (2009).

[52] Jasinski, J. and Liliental-Weber, Z. J. Electron. Mater. 31, 429 (2002).

[53] Lebedev, V., Cimalla, V., Pezoldt, J., Himmerlich, M., Krischok, S., Schaefer, J. A., Am- 
bacher, O., Morales, F. M., Lozano, J. G., and González, D. J. Appl. Phys. 100, 094902 (2006).

[54] Lebedev, V., Cimalla, V., Baumann, T., Ambacher, O., Morales, F. M., Lozano, J. G., and Gonzlez, D. J. Appl. Phys. 100, 094903 (2006).

[55] Wang, X., Che, S. B., Ishitani, Y., and Yoshikawa, A. Appl. Phys. Lett. 90, 151901 (2007).

[56] Cimalla, V., Lebedev, V., Morales, F. M., Goldhahn, R., and Ambacher, O. Appl. Phys. Lett. 89, 172109 (2006).

[57] Wang, H., Jiang, D. S., Wang, L. L., Sun, X., Liu, W. B., Zhao, D. G., Zhu, J. J., Liu, Z. S., Wang, Y. T., Zhang, S. M., and Yang, H. J. Phys. D: Appl. Phys. 41(13), 135403 (2008).

[58] Hsu, L., Jones, R. E., Li, S. X., Yu, K. M., and Walukiewicz, W. J. Appl. Phys. 102, 073705 (2007).

[59] Wang, X., Che, S. B., Ishitani, Y., and Yoshikawa, A. Appl. Phys. Lett. 92, 132108 (2008).

[60] Brown, G. F., Ager III, J. W., Walukiewicz, W., Schaff, W. J., and Wu, J. Applied Physics Letters 93, 262105 (2008).

[61] King, P. D. C., Veal, T. D., Gallinat, C. S., Koblmüller, G., Bailey, L. R., Speck, J. S., and McConville, C. F. Journal of Applied Physics 104, 103703 (2008).

[62] Arnaudov, B., Paskova, T., Paskov, P. P., Magnusson, B., Valcheva, E., Monemar, B., Lu, H., Schaff, W. J., Amano, H., and Akasaki, I. Physical Review B 69(11), 115216 (2004).

[63] Klochikhin, A. A., Davydov, V. Y., Emtsev, V. V., Sakharov, A. V., Kapitonov, V. A., Andreev, B. A., Lu, H., and Schaff, W. J. Phys. Rev. B 71(19), 195207 (2005).

[64] Inushima, T., Fukui, K., Lu, H., and Schaff, W. J. Appl. Phys. Lett. 92, 171905 (2008). 